\title{
High statistics determination of the strong coupling constant in Taylor scheme and its OPE Wilson coefficient from lattice QCD with a dynamical charm
}

\author{
B. Blossier, ${ }^{1}$ Ph. Boucaud, ${ }^{1}$ M. Brinet,${ }^{2}$ F. De Soto,${ }^{3}$ V. Morenas,${ }^{4}$ O. Pène,${ }^{1}$ K. Petrov,${ }^{5}$ and J. Rodríguez-Quintero ${ }^{6,7}$ \\ ${ }^{1}$ Laboratoire de Physique Théorique, Université de Paris XI; Bâtiment 210, 91405 Orsay Cedex; France \\ ${ }^{2}$ Laboratoire de Physique Subatomique et de Cosmologie, \\ CNRS/IN2P3/UJF; 53, avenue des Martyrs, 38026 Grenoble, France \\ ${ }^{3}$ Dpto. Sistemas Físicos, Químicos y Naturales, Univ. Pablo de Olavide, 41013 Sevilla, Spain \\ ${ }^{4}$ Laboratoire de Physique Corpusculaire, Université Blaise Pascal, CNRS/IN2P3 6317r Aubière Cedex, France \\ ${ }^{5}$ Laboratoire de l'Accélérateur Linéaire, Centre Scientifique d'Orsay; Bâtiment 200, 91898 ORSAY Cedex, France \\ ${ }^{6}$ Dpto. Física Aplicada, Fac. Ciencias Experimentales; Universidad de Huelva, 21071 Huelva; Spain. \\ ${ }^{7}$ CAFPE, Universidad de Granada, E-18071 Granada, Spain
}

\begin{abstract}
This paper reports on the determination of $\alpha_{S}$ from lattice simulations with $2+1+1$ twisted-mass dynamical flavours via the computation of the ghost-gluon coupling renormalized in the MOM Taylor scheme. A high-statistics sample of gauge configurations, used to evaluate the coupling from ghost and gluon propagators, allows for the appropriate update of previous results, now performing an improved analysis of data with reduced statistical errors and the systematical uncertainties under a better control.
\end{abstract}

PACS numbers: 12.38.Aw, 12.38.Lg

LPT-Orsay 13-77

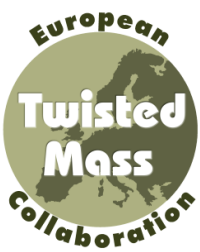

UHU-FT/13-09

\section{INTRODUCTION}

With QCD in our hands as the theory for the strong interaction, the running of the strong coupling constant can be computed by the perturbation theory in the UV domain, supplemented by nonperturbative OPE corrections when the IR domain is approached. Thus, values of the strong coupling constant obtained from experimental data at many different scales can be confronted to each others and to the theoretical running. This successful confrontation is a benchmark calculation of QCD. When integrated, the Renormalization Group equation which governs the running of the coupling constant introduces an energy scale, $\Lambda_{\mathrm{QCD}}$, as a boundary condition. $\Lambda_{\mathrm{QCD}}$ is defined for a given number of active quark species and can be only obtained as an input from experiment.

Lattice computations can be used for the purpose of deriving $\Lambda_{\mathrm{QCD}}$ from an experimental input. The usual procedure can be shortly described as follows. Any quantity extracted from a lattice simulation is obtained in unit of the lattice spacing, $a$. This scale depends on the bare coupling and others bare set-up parameters used in the simulation. Its value in physical unit is fixed by confronting a low-energy experimental number (a mass, a decay constant, ...) with its lattice prediction. Once the lattice spacing is known, the coupling at a given momentum can be computed from the lattice. Then, $\Lambda_{\mathrm{QCD}}$ can be subsequently derived from that coupling value. Among the methods most extensively applied to measure a coupling, one could find the implemention of the Schrödinger functional scheme (see, for instance, 114 and references therein), those based on the perturbative analysis of short-distance sensitive lattice observables as the inter-quark static potential (see for instance [5, 6]), the "boosted" lattice coupling (see [7-10] and references therein), moments of charmonia two-point correlation functions (see [11 13 and references therein) or, in particular, those based on the study of the momentum behaviour of Green functions (see [14 20], for instance).

Very recently, in refs. [21, 22, we reported on our preliminary studies of the strong coupling running and evaluation of $\Lambda_{\mathrm{QCD}}$ with lattice simulations including up, down, strange and charm dynamical quarks in the sea. These two were the last of a series of works (see also [23 26]) aimed at computing the strong coupling through the lattice determination of the ghost-gluon coupling in the so-called MOM Taylor renormalization scheme. A similar program developed in parallel by different authors [20, 27, 28, although following a different approach for the data analysis, is also in progress. The current paper is entirely devoted to complete this series of papers with upgraded results obtained 
by performing an improved analysis. The main ace for the improvement is the use of a new high-statistics sample of gauge configurations which reduces the statistical errors and allows for a better control of systematic uncertainties. In particular, we relax assumptions previously made for the $O(4)$-invariant lattice artefacts which can be fitted from the data. Such an improved analysis confirms first that nonperturbative power corrections are required for the running description of the data within our available momentum window. These power corrections, at the leading order ${ }^{1}$, appear to behave as predicted by SVZ sum-rules [32, 33. Then, we finally provide with a lattice estimate for $\Lambda_{\mathrm{QCD}}$ in good agreement with the current "world average" given by Particle Data Group [34].

\section{II. $\alpha_{S}\left(q^{2}\right)$ FROM THE GHOST-GLUON COUPLING}

Updating the determination of the strong running coupling from the ghost-gluon vertex in the MOM Taylor scheme reported in [21] is the main goal of this work. The details of the procedure, that will be hereupon outlined, have been profusely described in many previous works [22, 23, 25, 26, 35].

The keystone for an accurate determination of $\alpha_{S}\left(q^{2}\right)$ from the ghost-gluon coupling in the MOM Taylor scheme is the well-known Taylor's statement about the non-renormalization of the ghost-gluon vertex with vanishing incoming ghost momentum in Landau gauge [36. This implies that one can define a particular scheme for the QCD coupling such that its running with the renormalization momenta only relies on two-point Green functions renormalization constants [20, 23, 37,

$$
\alpha_{T}\left(\mu^{2}\right) \equiv \frac{g_{T}^{2}\left(\mu^{2}\right)}{4 \pi}=\lim _{\Lambda \rightarrow \infty} \frac{g_{0}^{2}\left(\Lambda^{2}\right)}{4 \pi} Z_{3}\left(\mu^{2}, \Lambda^{2}\right) \widetilde{Z}_{3}^{2}\left(\mu^{2}, \Lambda^{2}\right)
$$

where $Z_{3} / \widetilde{Z}_{3}$ stand for the gluon/ghost propagator renormalization constant and $\Lambda$ is a non specified UV cut-off. The main advantage resulting from this definition is that a very accurate nonperturbative estimate of the coupling and a very precise knowledge for its running from continuum perturbation tools can be both attained. It is worth to recall that an effective QCD charge definition, related to the pinching-technique effective charge 38 and useful for phenomenological purposes [39, can be grounded on this coupling in MOM Taylor scheme [40, 41. Eq. (1) being regularization independent, we will use lattice-regularized ghost and gluon propagators to estimate $\alpha_{T}$, provided that the lattice artefacts are properly kept under control such that the continuum limit is well recovered. The latter will be tested by considering, as will be seen below, different lattice set-up's and verifying that Eq. (1)'s results do not depend on the UV regularization cut-off, i.e. the lattice spacing.

\section{A. The running of $\alpha_{T}\left(q^{2}\right)$}

Specially crucial is the very precise knowledge for the running of $\alpha_{T}$ with momenta. At the four-loop level, from continuum perturbation theory, and with an Operator Product expansion (OPE) at the leading power correction, it reads 25$]$,

$$
\left.\alpha_{T}\left(\mu^{2}\right)=\alpha_{T}^{\text {pert }}\left(\mu^{2}\right)\left(1+\frac{9}{\mu^{2}} R\left(\alpha_{T}^{\text {pert }}\left(\mu^{2}\right), \alpha_{T}^{\text {pert }}\left(q_{0}^{2}\right)\right)\left(\frac{\alpha_{T}^{\text {pert }}\left(\mu^{2}\right)}{\alpha_{T}^{\text {pert }}\left(q_{0}^{2}\right)}\right)^{1-\gamma_{0}^{A^{2}} / \beta_{0}} \frac{g_{T}^{2}\left(q_{0}^{2}\right)\left\langle A^{2}\right\rangle_{R, q_{0}^{2}}}{4\left(N_{C}^{2}-1\right)}+\frac{1}{\mu^{2}}\right)\right)
$$

where $q_{0}$ is a renormalization point to be fixed, $\gamma_{0}^{A^{2}}$ can be taken from [42, 43] and gives for $N_{f}=4$,

$$
1-\gamma_{0}^{A^{2}} / \beta_{0}=\frac{27}{132-8 N_{f}}=\frac{27}{100} ;
$$

and, taking advantage of the $\overline{\mathrm{MS}}$ Wilson coefficients for the gluon and ghost OPE expansions at the $\mathcal{O}\left(\alpha^{4}\right)$-order $[43$, one can obtain in the appropriate renormalization scheme 35]

$$
R\left(\alpha, \alpha_{0}\right)=\left(1+1.03735 \alpha+1.07203 \alpha^{2}+1.59654 \alpha^{3}\right)\left(1-0.54993 \alpha_{0}-0.14352 \alpha_{0}^{2}-0.07339 \alpha_{0}^{3}\right)
$$

\footnotetext{
1 The OPE power corrections are found to be dominated by a non-vanishing landau-gauge dimension-two gluon condensate [17 29] 31
} 
to be plugged into Eq. (2). The purely perturbative running in Eq. (2) is given up to four-loops by [44]

$$
\begin{aligned}
\alpha_{T}^{\text {pert }}\left(\mu^{2}\right) & =\frac{4 \pi}{\beta_{0} t}\left(1-\frac{\beta_{1}}{\beta_{0}^{2}} \frac{\log (t)}{t}+\frac{\beta_{1}^{2}}{\beta_{0}^{4}} \frac{1}{t^{2}}\left(\left(\log (t)-\frac{1}{2}\right)^{2}+\frac{\widetilde{\beta}_{2} \beta_{0}}{\beta_{1}^{2}}-\frac{5}{4}\right)\right) \\
& +\frac{1}{\left(\beta_{0} t\right)^{4}}\left(\frac{\widetilde{\beta}_{3}}{2 \beta_{0}}+\frac{1}{2}\left(\frac{\beta_{1}}{\beta_{0}}\right)^{3}\left(-2 \log ^{3}(t)+5 \log ^{2}(t)+\left(4-6 \frac{\widetilde{\beta}_{2} \beta_{0}}{\beta_{1}^{2}}\right) \log (t)-1\right)\right)
\end{aligned}
$$

with $t=\ln \frac{\mu^{2}}{\Lambda_{T}^{2}}$ and the coefficients of the $\beta$-function in Taylor-scheme [45] (see also 23] where these coefficients have been shown to result from those for the ghost and gluon anomalous dimensions). The $\Lambda_{\mathrm{QCD}}$ parameters in Taylor-scheme and $\overline{\mathrm{MS}}$ are related through [25]

$$
\frac{\Lambda_{\overline{\mathrm{MS}}}}{\Lambda_{T}}=e^{-\frac{507-40 N_{f}}{792-48 N_{f}}}=0.560832,
$$

for the $\mathrm{N}_{f}=4$ case. Thus, an accurate determination of $\Lambda_{\overline{\mathrm{MS}}}$ and the $\overline{\mathrm{MS}}$ version of Eq. (5) allows for obtaining the standard $\overline{\mathrm{MS}}$ running coupling at any momentum.

The accurate determination of $\Lambda_{\overline{\mathrm{MS}}}$ will result, as will be seen below, from the confrontation of Eq. (2) with a lattice estimate of Eq. (1) within the appropriate momenta window. The ghost and gluon renormalization constants to be here applied in Eq. (1) have been obtained from $N_{f}=2+1+1$ gauge configurations for several bare couplings, light twisted masses and volumes. This allows for a thorough study of the artefacts from the lattice regularization and the required chiral extrapolation. The first type of artefacts affecting the measured quantity $\alpha_{T}^{\text {Latt }}$ concerns the breaking of the full $O(4)$ rotational symmetry. The the so-called $H(4)$-extrapolation procedure [46 48] exploits the remaining $H(4)$ isometry group symmetry and the discrepancies within $H(4)$ orbits, which at small enough momenta can be written as :

$$
\alpha_{T}^{\mathrm{Latt}}\left(a^{2} q^{2}, a^{2} \frac{q^{[4]}}{q^{2}}, \ldots\right)=\widehat{\alpha}_{T}\left(a^{2} q^{2}\right)+\left.\frac{\partial \alpha_{T}^{\mathrm{Latt}}}{\partial\left(a^{2} \frac{q^{[4]}}{q^{2}}\right)}\right|_{a^{2} \frac{q^{[4]}}{q^{2}}=0} a^{2} \frac{q^{[4]}}{q^{2}}+\ldots
$$

where $q^{[4]}=\sum_{i} q_{i}^{4}$ is the first relevant $H(4)$-invariant, to cure these effects (see appendix A). Then $\widehat{\alpha}_{T}$ is written as :

$$
\widehat{\alpha}_{T}\left(a^{2} q^{2}\right)=\alpha_{T}\left(q^{2}\right)+c_{a 2 p 2} a^{2} q^{2}+o\left(a^{2}\right),
$$

which can be used to fit and eliminate the dominant $O(4)$-invariant artefact from the lattice estimates 49 [1]. One is then left with the continuum prediction, $\alpha_{T}$, to be confronted with Eq. (2).

\section{B. The lattice data}

Then, Eqs. 2 2 87 can be used to predict the running of $\alpha_{T}\left(q^{2}\right)$ for a momenta window where OPE higher-powers, $o\left(1 / q^{2}\right)$, and $o\left(a^{2} q^{2}\right)$ lattice artefacts could be neglected. Within such a window, the only three parameters, $g^{2}\left\langle A^{2}\right\rangle$, $\Lambda_{\overline{\mathrm{MS}}}$ and the coefficient for the $O(4)$-invariant artefacts $c_{a 2 p 2}$, remain free to be fitted to account for the lattice estimate of the Taylor coupling through Eq. (1). Aiming at obtaining $\alpha_{T}^{\mathrm{Latt}}$ by Eq. (1), the ghost and gluon propagators are computed from the gauge configurations simulated at several lattices with $N_{f}=2+1+1$ twisted-mass lattice flavors [52] by the ETM collaboration [53, 54. In the gauge sector, we use the Iwasaki action and compute the propagators as described in refs. [25, while for the fermion action we have

$$
S_{l}=a^{4} \sum_{x} \bar{\chi}_{l}(x)\left(D_{W}[U]+m_{0, l}+i \mu_{l} \gamma_{5} \tau_{3}\right) \chi_{l}(x)
$$

for the doublet of degenerate light quarks [55] and

$$
S_{h}=a^{4} \sum_{x} \bar{\chi}_{h}(x)\left(D_{W}[U]+m_{0, h}+i \mu_{\sigma} \gamma_{5} \tau_{1}+\mu_{\delta} \tau_{3}\right) \chi_{h}(x)
$$

for the heavy doublet. $D_{W}[U]$ is the standard massless Wilson Dirac operator. The lattice parameters for the ensembles of gauge configurations we used are given in tab. I Tuning to maximal twist is achieved by choosing a 
parity odd operator and determining $\kappa_{\text {crit }}$ such that this operator has a vanishing expectation value. One appropriate quantity is the PCAC light quark mass and we demand $m_{P C A C}=0$. We refer the interested reader to refs. [53, 54] for more details about the set-up of the twisted mass lattice simulations. The results for $\widehat{\alpha}_{T}$ obtained, as explained, with Eq. (1) from the lattice data for the ensembles in Tab. I appear plotted in Fig. 1. The errors have been estimated through the usual jackknife method, by applying the $H(4)$-extrapolation procedure, explained above, to the estimates of $\widehat{\alpha}_{T}$ from each jackknife cluster of configurations we made. As can be seen, this $H(4)$-extrapolation works pretty well and very smooth curves, with no trace of the usual oscillations from the $O(4)$ breaking, are obtained.

\begin{tabular}{||c|c|c|c|c|c|c||}
\hline$\beta$ & $\kappa_{\text {crit }}$ & $a \mu_{l}$ & $a \mu_{\sigma}$ & $a \mu_{\delta}$ & $(L / a)^{3} \times T / a$ & confs. \\
\hline 1.90 & 0.163267 & 0.0050 & 0.150 & 0.190 & $32^{3} \times 64$ & 500 \\
& 0.163270 & 0.0040 & & & $32^{3} \times 64$ & 500 \\
& 0.163272 & 0.0030 & & & $32^{3} \times 64$ & 500 \\
\hline 2.1 & 0.156357 & 0.0020 & 0.120 & 0.1385 & $48^{3} \times 96$ & 800 \\
\hline
\end{tabular}

TABLE I: Lattice set-up parameters for the ensembles we used in this paper. The last column stands for the number of gauge field configurations we used.

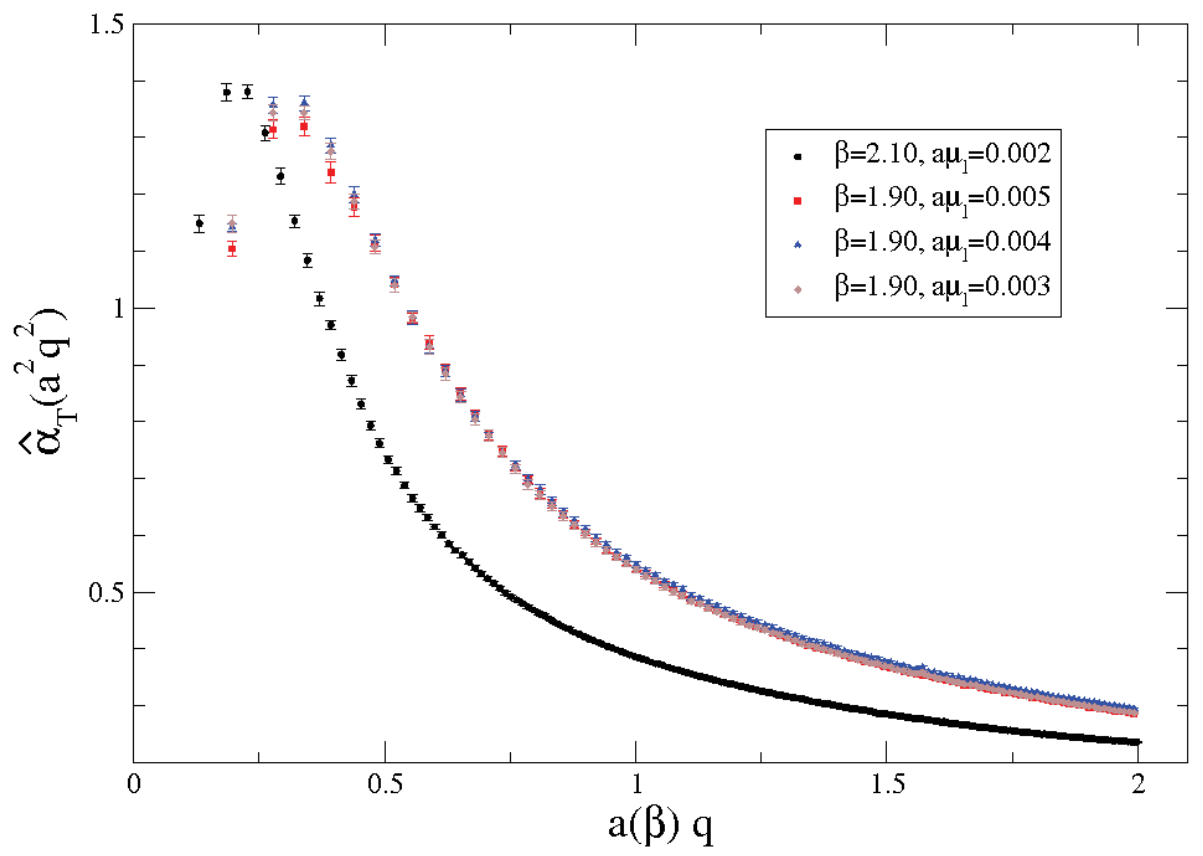

FIG. 1: Lattice estimates for $\hat{\alpha}_{T}$ from the gluon and ghost propagators obtained with the ensembles of Tab. I] applied first in Eq. (1) and cured then for the $H(4)$-invariant hypercubic artefacts as explained around Eq. (7). They appear plotted in terms of the lattice momenta, $a(\beta) q$, and a first cut, $a(\beta) q<2$, is applied to avoid large hypercubic artefacts.

\section{ANALYSING THE DATA}

The first step in our strategy for analysing the data is to exploit the high statistics (800 gauge configurations) for the lattice ensemble at $\beta=2.10$ (see Tab. I) in order to extract precisely the physical running of $\alpha_{T}\left(q^{2}\right.$ ). Eqs. (2) 8 , obtained from continuum perturbation theory and OPE analysis, are thought to describe the running behaviour of the lattice estimate for the Taylor coupling through Eq. (1), only up to $o\left(1 / q^{2}\right)$ higher-power OPE corrections and $o\left(a^{2} q^{2}\right)$ lattice artefacts. Then, in order to apply Eqs. (2) and determine its three free-parameters by a fit, one needs to identify the appropriate window of momenta, large enough for the running behaviour not to be polluted by higher OPE powers but small enough as not to be affected by higher-order discretization artefacts. As far as we are dealing with only one lattice ensemble, at given bare coupling $(\beta)$ and quark masses $\left(a \mu_{l}, a \mu_{\sigma}, a \mu_{\delta}\right)$, we can skip the problem of the absolute lattice calibration (the determination of the lattice spacing, $a(\beta)$, in physical units) that 
can be put back to a final stage of the analysis. Consequently, all the fitted dimensionful parameters will be now expressed in units of the lattice spacing and, in particular, the perturbative running given by Eq. (5) will depend on $t=\ln \left(a^{2}(\beta) q^{2} / a^{2}(\beta) \Lambda_{T}^{2}\right)$.

\section{A. The optimal window fit}

We first perform a fit, for every set of data with lattice momenta above $a(\beta) q \simeq 1.15$, whose results read as in Tab. II. Then, the upper and lower bounds for the fitting window have been systematically shifted, the window size roughly ranging from 0.4 to 0.8 in units of $a(2.10)^{-1}$, to look for the optimal fit (the one with the minimum value of $\chi^{2}$ d.o.f.). This optimal fit is found to happen for data with lattice momenta within $1.31<a(\beta) q<1.81$ (see Tab. II . In both cases, the renormalization point for Eq. (2) is $\operatorname{chosen}^{2}$ to be $a(2.10) q_{0}=2.92$.

\begin{tabular}{||c|c|c|c|c||}
\hline fit window & $\chi^{2} /$ d.o.f. & $\Lambda_{\overline{\mathrm{MS}}} a(2.1)$ & $g^{2}\left\langle A^{2}\right\rangle a^{2}(2.1)$ & $c_{\mathrm{a} 2 \mathrm{p} 2}$ \\
\hline$[1.15,2.0]$ & 0.864 & $0.092(5)$ & $0.39(11)$ & $-0.0049(8)$ \\
\hline$[1.31,1.81]$ & 0.270 & $0.099(3)$ & $0.26(7)$ & $-0.0066(4)$ \\
\hline
\end{tabular}

TABLE II: Fitting parameters expressed in units of $a^{-1}(2.1)$ for the two different fitting windows discussed in the text: all momenta larger than $a(2.1) q>1.15$ (first line) and the window for the optimal fit (second line). The errors reported here are statistical ones, obtained from the jackknife's method.

Furthermore, according to Eq. (2),

$$
\left(\frac{\alpha_{T}\left(q^{2}\right)}{\alpha_{T}^{\text {pert }}\left(q^{2}\right)}-1\right) q^{2}=9 R\left(\alpha_{T}^{\text {pert }}\left(q^{2}\right), \alpha_{T}^{\text {pert }}\left(q_{0}^{2}\right)\right)\left(\frac{\alpha_{T}^{\text {pert }}\left(q^{2}\right)}{\alpha_{T}^{\text {pert }}\left(q_{0}^{2}\right)}\right)^{1-\gamma_{0}^{A^{2} / \beta_{0}}} \frac{g_{T}^{2}\left(q_{0}^{2}\right)\left\langle A^{2}\right\rangle_{R, q_{0}^{2}}}{4\left(N_{C}^{2}-1\right)}
$$

up to terms vanishing at large $q^{2}$. Then, Eq. 111)'s l.h.s. can be computed with $\alpha_{T}$ from the lattice through Eq. (8) and its perturbative four-loop prediction from Eq. (5), with the best-fit parameters for $\Lambda_{\overline{\mathrm{MS}}}$ from Tab. II. As Eq. (11)'s r.h.s. reads, this, plotted in terms of momenta in lattice units within the appropriate range, makes essentially the running of the Wilson coefficient to appear. This can be seen in Fig. 2; a clearly nonzero nonperturbative contribution appears remarkably to behave as the OPE analysis predicts ${ }^{3}$, although some systematic deviation for the data can be noticed from the expected behaviour in the case of the fit over every momenta above $a(2.1) q>1.15$. The drastic diminution of $\chi^{2} /$ d.o.f. when the fit is restricted to the optimal window gives also a strong indication that, within that window, higher-order OPE corrections and discretization artefacts can be properly neglected.

\section{B. Higher-order power corrections}

The Taylor coupling estimated from the lattice should feel at low momenta the impact of nonpertubative contributions other than the leading OPE ones included in Eq. 22. Indeed, lattice data deviations from this OPE formula are clearly visible for momenta $a(2.10) q \lesssim 1.2$ in fig. 2 With the OPE machinery at hand, corrections to Eq. 2 , should be incorporated through condensates of higher-order local operators and appear thus suppressed by powers of the inverse of momentum higher than 2. Aiming at identifying the dominant next-to-leading contribution, lattice data deviations from Eq. (2) are plotted in fig. 3 in terms of the momenta, using for both axes logarithmic scales. As can be seen, a logarithmic slope of $\sim 6.15$ results from lattice data, after the subtraction of Eq. (2) with the best-fit parameters for the small window in Tab. II for momenta above $a(2.10) q \sim 0.5$ and below $a(2.10) q \sim 1.3$.

This logarithmic slope is consistent with the results we obtained in refs. [21, 22, where a $1 / p^{6}$-correction is incorporated to describe successfully the lattice data all over a large momenta window roughly ranging from $1.75 \mathrm{GeV}$ up to $7 \mathrm{GeV}$. As was discussed therein, such a $1 / p^{6}$-correction might not be necessarily explained by the dominance of a condensate of dimension six but might be an effective power originated either by the interplay of a next-to-leading

\footnotetext{
2 This choice would imply, had we taken the lattice spacing for $\beta=2.10$ used in ref. [22, $q_{0}=10 \mathrm{GeV}$.

3 A similar analysis has been also preliminary performed in 22] where, with a poorer statistics for the lattice ensembles (50 gauge configurations), the Wilson coefficient running has also been proven to be required in order to properly describe the lattice data of $\alpha_{T}$ for physical momenta above around $4 \mathrm{GeV}$.
} 


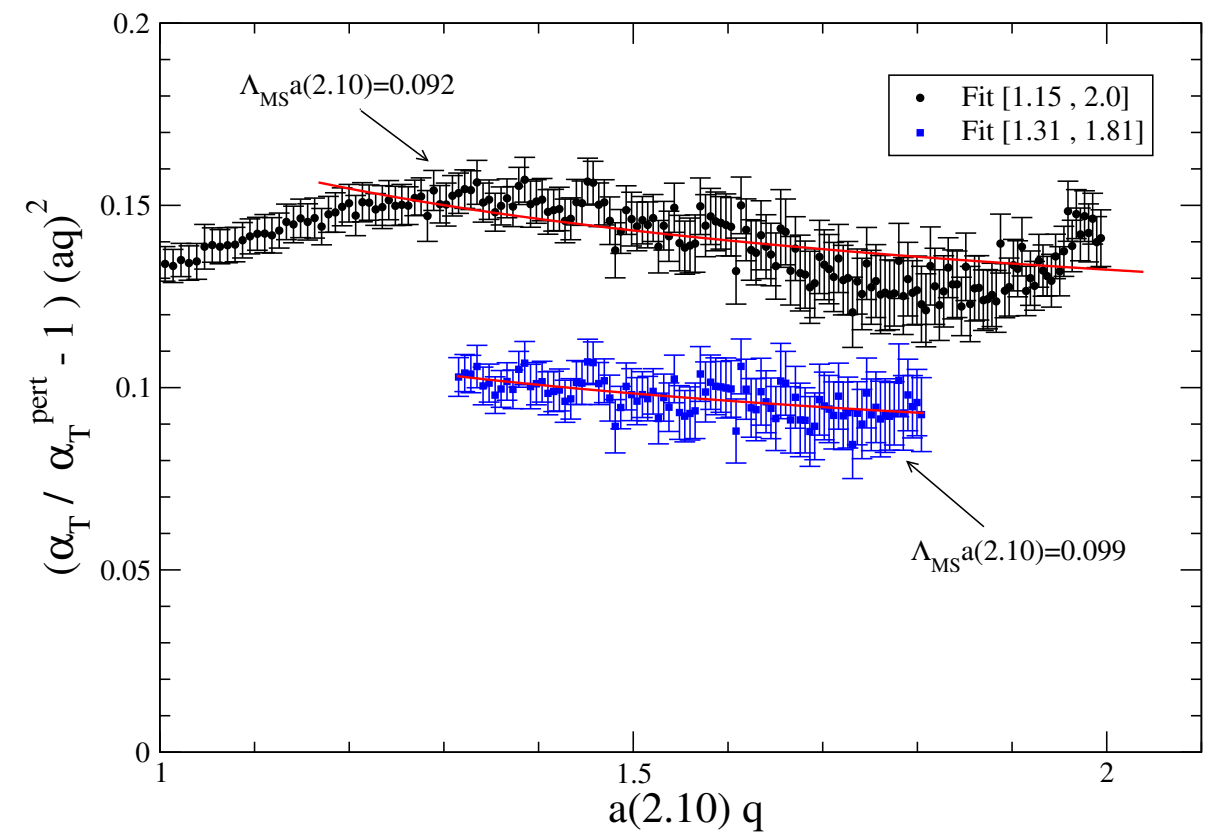

FIG. 2: Eq. 11]'s 1.h.s. estimated with $\alpha_{T}$ from the lattice data for the ensemble at $\beta=2.10$ (800 confs.) and its fourloop perturbative prediction evaluated with $\Lambda_{\overline{\mathrm{MS}}}$ from Tab. II The solid red line corresponds to the evaluation of Eq. 11 's r.h.s., also with the best-fit parameter for $g^{2}\left\langle A^{2}\right\rangle$ in Tab. II All the dimensionful parameters should be taken in units of the appropriate lattice spacing power.

power correction and its Wilson coefficient or by a different nonperturbative mechanism being dominant at these low-momenta. Anyhow, on the ground of the striking result shown by Fig. 3, one can firmly argue that Eq. (2) with the addition of a $1 / p^{x}$-contribution, where $x$ is around 6 , should account for the running of lattice data also at very low momenta. Thus, Eq. (8) could be replaced by

$$
\widehat{\alpha}_{T}\left(a^{2} q^{2}\right)=\alpha_{T}^{\mathrm{A} 2}\left(q^{2}\right)+\frac{d_{x}}{q^{x}}+c_{a 2 p 2} a^{2} q^{2}+o\left(a^{2}\right),
$$

where the physical running of the coupling is now given by

$$
\alpha_{T}\left(q^{2}\right)=\alpha_{T}^{A 2}\left(q^{2}\right)+\frac{d_{x}}{q^{x}}
$$

$\alpha_{T}^{A 2}$ being still given by Eq. 20.

We can now try to fit Eq. (12) to the lattice estimates for the coupling through Eq. (1), for all momenta such that $a(2.10) q>0.5$. However, if one keeps $c_{a 2 p 2}, \Lambda_{\overline{\mathrm{MS}}}, g^{2}\left\langle A^{2}\right\rangle, d_{x}$ and the power $x$ as free parameters, all of them appear strongly correlated allowing for inconsistent values of $x$, with unnaturally large gluon condensates and much lower values of $\Lambda_{\overline{\mathrm{MS}}}$. Thus, as one can judiciously guess that higher-order power corrections may appear naturally correlated with the leading OPE contribution but should not affect too much the parameter driving the purely perturbative running, $\Lambda_{\overline{\mathrm{MS}}}$, we will apply the following two-step procedure: (i) first, we will take for $\Lambda_{\overline{\mathrm{MS}}}$ and $c_{a 2 p 2}$ the values from Tab. II and fit Eq. (12) to the data with $g^{2}\left\langle A^{2}\right\rangle, d_{x}$ and $x$ as free parameters; (ii) then, we will assume $x$, for the higher-order power corrections, to be well determined by the previous step and perform a new fit of Eq. (12) to the data with the other four free parameters. The results are gathered in Tab. [III It should be noticed that a power value compatible with 6 within errors is found for the higher-order power correction and that the best-fit parameters here obtained and those in the previous section (without including higher-order corrections, but shifting the window lower bound up to a larger momentum) are also compatible within the errors. 


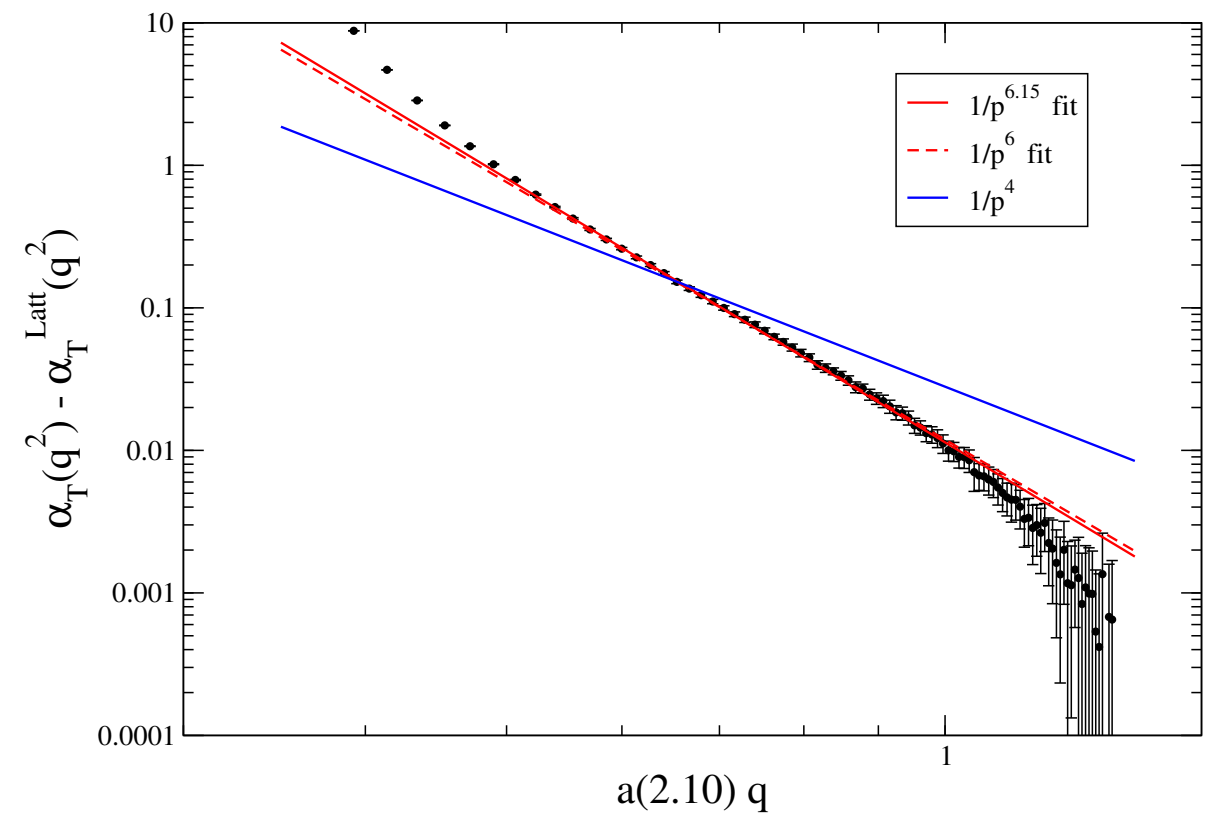

FIG. 3: Lattice data deviations from Eq. 2) with the best-fit parameters for the small window in Tab. II] plotted in terms of the momentum in units of the lattice spacing. Logarithmic scales are applied for the coordinates in both axes. A best-fit for the logarithmic slope of 6.15 is shown in red solid line as well as, for the sake of comparison, fits with slopes fixed to be 6 (red dashed) and 4 (blue).

\begin{tabular}{|c|c|c|c|c|c|}
\hline & $c_{a 2 p 2}$ & $\Lambda_{\overline{\mathrm{MS}}} a(2.10)$ & $g^{2}\left\langle A^{2}\right\rangle a^{2}(2.10)$ & $d_{x} a^{x}(2.10)$ & $x$ \\
\hline step (i) & & & $0.29(2)$ & $-0.0159(22)$ & $5.73(27)$ \\
\hline step (ii) & $-0.0058(4)$ & $0.0957(22)$ & $0.35(5)$ & $-0.0168(11)$ & \\
\hline
\end{tabular}

TABLE III: Results obtained with the two-steps procedure, explained in the text, followed to account with Eq. 12 for the Taylor coupling computed with the lattice at $\beta=2.10$. The bold-face characters indicate the best-fit parameters that will be applied in the following to describe data for any momenta above $a(2.10) q>0.5$.

\section{Higher-order lattice artefacts and the running of the Wilson coefficient}

According to Eq. (12), one can define

$$
\Delta\left(a^{2} q^{2}\right)=\widehat{\alpha}_{T}\left(a^{2} q^{2}\right)-\alpha_{T}^{A 2}\left(q^{2}\right)-\frac{d_{x}}{q^{x}}=c_{a 2 p 2} a^{2} q^{2}+o\left(a^{2}\right)
$$

that, up to $o\left(a^{2}\right)$-corrections, should behave linearly on $a^{2} q^{2}$. Indeed, we have found this to happen for $\Delta\left(a^{2} q^{2}\right)$ computed by subtracting $\alpha_{T}^{A 2}\left(q^{2}\right)$ from Eq. (2) and the higher-order power correction, with the best-fit parameters (in bold-faced characters) of Tab. III. to the coupling lattice data. The latter can be seen in Fig. 4. Furthermore, very small oscillatory deviations from the linear behaviour can be appreciated (bottom plot of Fig. 44). This might imply for the $O(4)$-invariant lattice artefacts a more complicate structure, always behaving as $a^{2} q^{2}$ in a first order Taylor expansion, or a relic of $O(4)$-breaking artefacts not totally cured with the $H(4)$-extrapolation procedure ${ }^{4}$. In practice, such a small deviation could be in practice pretty well parameterized by $r_{0} a q \sin \left(r_{1} a q\right)$, as can be seen in Fig. 4

A final remark is now in order: after the removal of lattice artefacts and the higher-order power correction, the running of the leading OPE Wilson coefficient can be now properly shown over a large window, $a(2.10) q>0.5$. In

\footnotetext{
4 To remove $O(4)$-breaking artefacts at the order $O\left(a^{2}\right)$, one just needs to extrapolate $a^{2} q^{[4]} / q^{2}$ to 0 . To go beyond, apart from $a^{4}\left(q^{[4]} / q^{2}\right)^{2}$, one would need to include $a^{4} q^{[6]} / q^{2}$ terms in the extrapolation which would so increase the difficulty of the computation.
} 

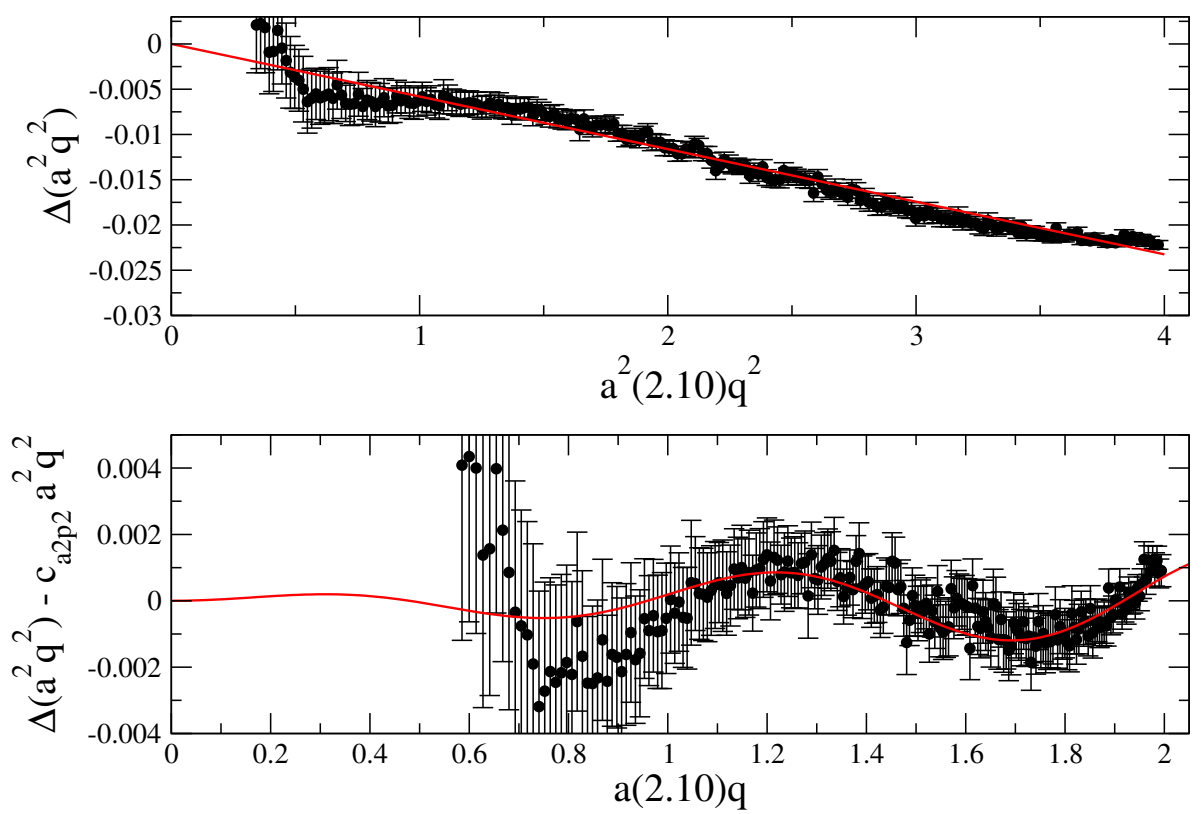

FIG. 4: (Top) $\Delta\left(a^{2} q^{2}\right)$ computed through Eq. 14 from lattice data after the subtraction of the running given by Eq. 22 and the higher-order power correction. It is shown to behave as one expects due to $O(4)$-invariant leading artefacts (solid red line). Very small oscillatory deviations from the leading behaviour can be appreciated and appear plotted in terms of $a q$ (bottom) which can be fitted in practice by $r_{0} a q \sin \left(r_{1} a q\right)$ with $r_{0}=0.00071$ and $r_{1}=2.081 \pi$ (solid red line).

other words, Eq. (11)'s l.h.s. can be now plotted in terms of $a q$ with $\alpha_{T}$ replaced by $\alpha_{T}^{A 2}$ obtained from the lattice data through Eq. (12) and compared with the theoretical expression given in Eq. (11)'s rhs. This is done in the plot of Fig. 5, where the predicted running for the leading OPE Wilson coefficient is beautifully followed by the corrected lattice estimates for momenta above $a(2.10) q \sim 0.5$.

\section{SCALING AND LATTICE CALIBRATION}

We have focussed until now the analysis in exploiting the high-statistics ensemble of data obtained for a $48^{3} \times 96$ lattice at $\beta=2.10$ (see Tab. II). We thus obtained the results collected in Tab. II] when fitting with Eqs. (28), and in Tab. III, when applying Eqs. (2 12) including also a higher-order power correction. From now on, we can take advantage of the "scaling" for the running of the physical coupling at different $\beta$ 's in order to extract useful information, and perform a demanding crosscheck, from the three other ensembles of data at $\beta=1.90$ and different light masses (see Tab. I).

\section{A. Scaling at different $\beta$ 's}

The running coupling is a renormalized quantity not depending on either the regularization scheme or the regularization cut-off. After being properly cured for lattice artefacts, the resulting coupling expressed in terms of the physical momentum should not depend on $\beta$ and, one can judiciously argue, only slightly on the mass of the active quark flavours, provided that the momentum runs far enough away from the quark mass thresholds ${ }^{5}$. We will assume any flavour mass dependence to have been properly captured by the lattice spacing and thus write

$$
\widehat{\alpha}_{T}^{\left(\beta, \mu_{l}\right)}\left(a^{2}\left(\beta, \mu_{l}\right) q^{2}\right)-c_{a 2 p 2} a^{2}\left(\beta, \mu_{l}\right) q^{2} \equiv \widehat{\alpha}_{T}^{\left(\beta_{0}, \mu_{l}^{0}\right)}\left(a^{2}\left(\beta_{0}, \mu_{l}^{0}\right) q^{2}\right)-c_{a 2 p 2} a^{2}\left(\beta_{0}, \mu_{l}^{0}\right) q^{2},
$$

\footnotetext{
${ }^{5}$ In the standard $\overline{\mathrm{MS}}$ approach, the flavours become active at momenta above their $\overline{\mathrm{MS}}$ running quark masses, with the appropriate radiative corrections included in the matching formula [34, 56, 57.
} 


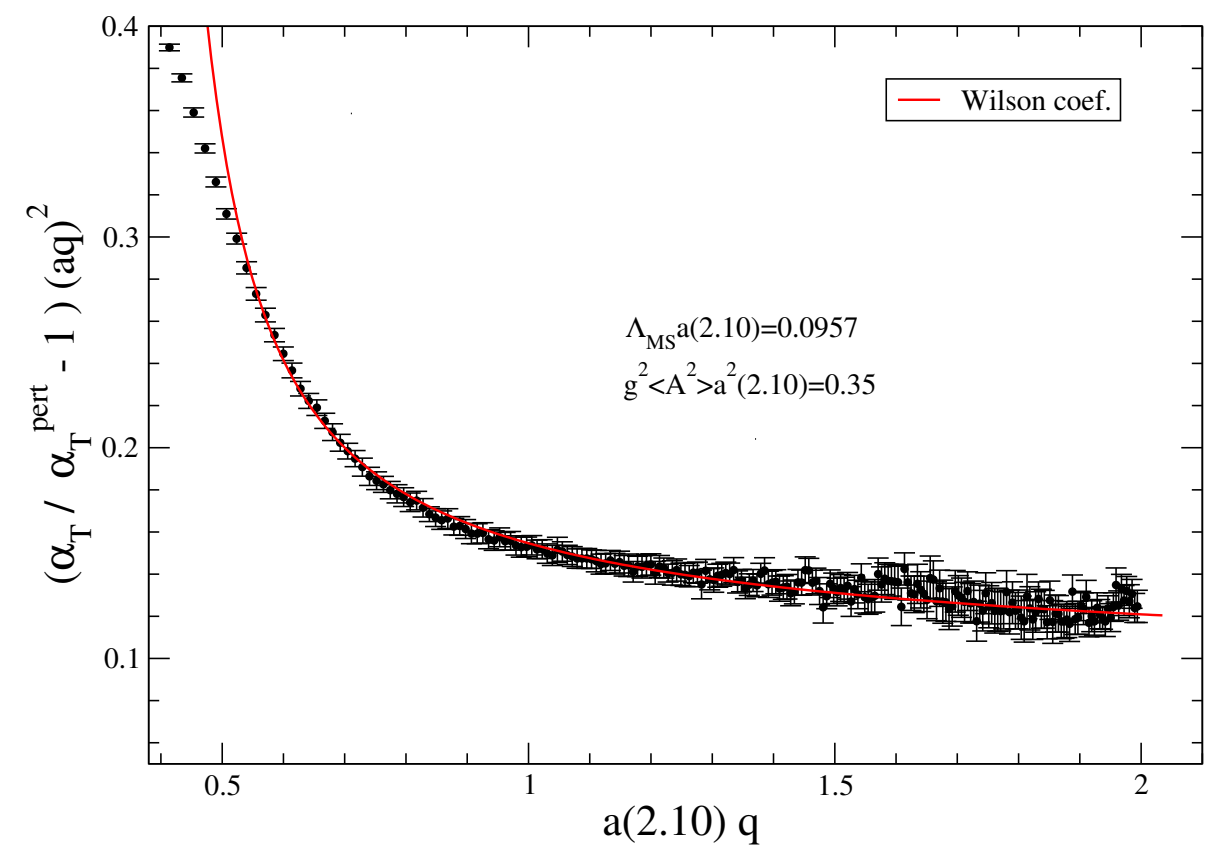

FIG. 5: The same as plotted in Fig. 2 but $\alpha_{T}$ in Eq. (11)'s l.h.s. is here evaluated only after dropping completely the lattice artefacts and the higher-order power correction away from the lattice data. The solid red line corresponds to the running predicted for the Wilson coefficient of the leading OPE power correction.

for any two bare coupling parameters, $\beta$ and $\beta_{0}$, and light flavour masses, $\mu_{l}$ and $\mu_{l}^{0}$ (we will neglect the effect of the heavy flavour masses). Eq. (15) stands for the explicit expression of the scaling condition for estimates from two different simulations at the same momentum $q$ in physical units. Now, we take $\beta_{0}=2.10$ and $\mu_{l}^{0}=0.002$ and, according to Eq. (12), replace Eq. (15)'s r.h.s. by the continuum running of $\alpha_{T}$ given by Eq. (2) and the higher power correction,

$$
\begin{aligned}
\widehat{\alpha}_{T}^{\left(\beta, \mu_{l}\right)}\left(\left(\frac{a\left(\beta, \mu_{l}\right)}{a\left(\beta_{0}, \mu_{l}^{0}\right)}\right)^{2} k_{L}^{2}\right)-c_{a 2 p 2}\left(\frac{a\left(\beta, \mu_{l}\right)}{a\left(\beta_{0}, \mu_{l}^{0}\right)}\right)^{2} k_{L}^{2} & =\alpha_{T}\left(k_{L}^{2} ; \Lambda_{\overline{\mathrm{MS}}} a\left(\beta_{0}, \mu_{l}^{0}\right), g^{2}\left\langle A^{2}\right\rangle a^{2}\left(\beta_{0}, \mu_{l}^{0}\right)\right) \\
& +\frac{d_{x}}{\left(a\left(\beta_{0}, \mu_{l}^{0}\right) k_{L}\right)^{x}}
\end{aligned}
$$

where $k_{L}=a\left(\beta_{0}, \mu_{l}^{0}\right) q$ stands for the momentum in units of the lattice spacing at $\beta_{0}$ and $\mu_{l}^{0}$. In Eq. (16)'s r.h.s., we write down explicitly the parameters $\Lambda_{\overline{\mathrm{MS}}}$ and $g^{2}\left\langle A^{2}\right\rangle$ used for $\alpha_{T}$ to specify that they are taken from Tab. III] also in units of the lattice spacing. It should be recalled that Eqs. (2 12$)$ are only suitable for describing the lattice data at $\beta=2.10$ when $k_{L}>0.5$. Then, this will be also the case for Eq. (16), although threshold mass effects might not be still negligible at $k_{L} \sim 0.5$.

Eq. (16)'s r.h.s. is completely determined by Eq. (2) and the best-fit parameters of Tab. III. Concerning its l.h.s., $\widehat{\alpha}_{T}$ can be obtained from a lattice simulation at any $\beta$ and $\mu_{l}$, after properly shifting the lattice momentum $a q$ through multiplication by the ratio of lattice spacings $a\left(\beta_{0}, \mu_{l}^{0}\right) / a\left(\beta, \mu_{l}\right)$, being so left with the physical momentum expressed in units of $a^{-1}\left(\beta_{0}, \mu_{l}^{0}\right), k_{L}$. Therefore, the ratio $a\left(\beta_{0}, \mu_{l}^{0}\right) / a\left(\beta, \mu_{l}\right)$ and $c_{a 2 p 2}$ can be obtained by requiring the data from l.h.s. to be best fitted by the r.h.s., for any $k_{L}>0.5$, with the minimum- $\chi^{2}$ criterion. The coefficient $c_{a 2 p 2}$ is treated as a free parameter although, by dimensional arguments from Eqs. 8 [12), it could be thought not to depend on the lattice spacing and, hence, not to depend on $\beta$ and $\mu_{l}$ either. However, as $c_{a 2 p 2}$ is to be determined by a fit of Eq. (12) to lattice data, it might borrow something from higher-order lattice artefacts and its fitted values might slightly depend on the lattice parameters for the simulation. The procedure has been followed for the analysis of data from the three ensembles at $\beta=1.90$ and the fit results have been shown in Tab. IV] As can be seen there, we are left with high-quality fits where, as expected, the coefficients $c_{a 2 p 2}$ are fully compatible with each other and, together with those obtained for $\beta=2.10$ in the previous section, all happen to lie fairly in the same ballpark. In previous analysis [21, 22, we imposed $c_{a 2 p 2}$ to be the same for different simulations at any $\beta$ and $\mu_{l}$, mainly in order to stabilize the fits; here we found this to happen within a reasonable degree of approximation which is compatible 
with assuming the extraction for $c_{a 2 p 2}$ to be polluted by higher-order artefacts. This strongly supports the approach followed in refs. [21, 22] and confirms the reliability of previous and present results.

\begin{tabular}{|c|c|c|c|}
\hline$\mu_{l}$ & $a(2.10,0.002) / a\left(1.90, \mu_{l}\right)$ & $c_{a 2 p 2}$ & $\chi^{2} /$ d.o.f. \\
\hline 0.003 & $0.6798(74)$ & $-0.0076(6)$ & $20.2 / 82$ \\
\hline 0.004 & $0.6683(72)$ & $-0.0067(6)$ & $14.4 / 82$ \\
\hline 0.005 & $0.6775(73)$ & $-0.0081(6)$ & $45.7 / 82$ \\
\hline
\end{tabular}

TABLE IV: Best-fit parameters obtained with Eq. (16) and the data for the three ensembles at $\beta=1.90$. The fitting momenta window is defined by $k_{L}>0.5$, as explained in the text.

\section{B. Chiral extrapolation and lattice calibration}

In the previous subsection, we have obtained the ratio of the lattice spacing for the simulations at $\beta=1.90$ and three different dynamical flavour masses, $a\left(1.90, \mu_{l}\right)$, over the spacing for the simulation at $\beta=2.10$ and $\mu_{l}=0.002$ (see Tab. IV]. Since the twisted-mass fermion action has been tuned to the maximal twist angle, the flavour mass happens to be proportional to $\mu_{l}$. On the other hand, all the flavour mass dependence is assumed to be captured by the lattice spacing which should be expected to behave linearly on $a^{2} \mu_{l}^{2}$ since the lattice action is improved at the $\mathcal{O}(a)$-order. One can thus write:

$$
\frac{a(2.10,0.002)}{a\left(\beta, \mu_{l}\right)}=\frac{a(2.10,0.002)}{a(\beta, 0)}\left(1+c_{\mu} a^{2}(\beta, 0) \mu_{l}^{2}+o\left(a^{2} \mu_{l}^{2}\right)\right),
$$

according to which, the ratios from Tab. IV can be extrapolated to reach the chiral limit, when the light flavour mass is exactly put to zero. Thus, as can be seen in Fig. 6. we obtain a remarkably weak slope:

$$
c_{\mu}=-1.1(7.8) \times 10^{2},
$$

clearly compatible wity zero, and

$$
a(2.10,0.002)=0.677(13) a(1.90,0)=0.0599(27) \mathrm{fm} ;
$$

where we applied the very recent result $a(1.90,0)=0.0885(36) \mathrm{fm}[58$, obtained by the ETM collaboration through chiral fits for the lattice pseudoscalar masses and decay constants that are required to take the experimental $f_{\pi}$ and $m_{\pi}$ at the extrapolated physical point. The pion masses for the simulations happen to range from 270 to $510 \mathrm{MeV}$.

Now, Eq. (19) can be used to make a conversion of our results to physical units and, together with the ratios of Tab. IV. the lattice estimates of $\widehat{\alpha}_{T}$ from simulations at $\beta=2.10$ and $\beta=1.90$ of Fig. 1 can be cured for $O(4)$-invariant lattice artefacts from Eq. (12) and plotted in terms of the momentum in physical units, where the evidence for the scaling expressed by Eq. $\sqrt{15})$ is to be weighted through the superposition of data from different simulations. This can be seen in Fig. 7, while the fit results in physical units are reported in Tab. V.

\begin{tabular}{||c|c|c|c||}
\hline fit window $[\mathrm{GeV}]$ & $\Lambda_{\overline{\mathrm{MS}}}[\mathrm{MeV}]$ & $g^{2}\left\langle A^{2}\right\rangle\left[\mathrm{GeV}^{2}\right]$ & $\left(-d_{x}\right)^{1 / x}[\mathrm{GeV}]$ \\
\hline \hline$[4.3,6.0]$ & $324(18)$ & $2.8(8)$ & \\
\hline$[1.7,6.6]$ & $314(16)$ & $3.8(6)$ & $1.61(7)$ \\
\hline
\end{tabular}

TABLE V: Fitted parameters from previous sections, now expressed in physical units with the help of Eq. 19p. The error estimates include the uncertainty on the lattice spacing determination given in Eq. [19.

\section{V. $\alpha_{\overline{\mathrm{MS}}}\left(q^{2}\right)$ FROM THE GHOST-GLUON COUPLING}

The $\overline{\mathrm{MS}}$ running coupling can be obtained by the integration of the $\beta$-function, with the coefficients now in $\overline{\mathrm{MS}}$ scheme, and $\Lambda_{\overline{\mathrm{MS}}}$ both for $\mathrm{N}_{f}=4$. We will read $\Lambda_{\overline{\mathrm{MS}}}$ from Tab. $\mathrm{V}$.

$$
\Lambda_{\overline{\mathrm{MS}}}=314(7)(14)(\mathbf{1 0}) \times\left(\frac{0.0599 \mathrm{fm}}{a(2.10,0.002)}\right) \mathrm{MeV}
$$




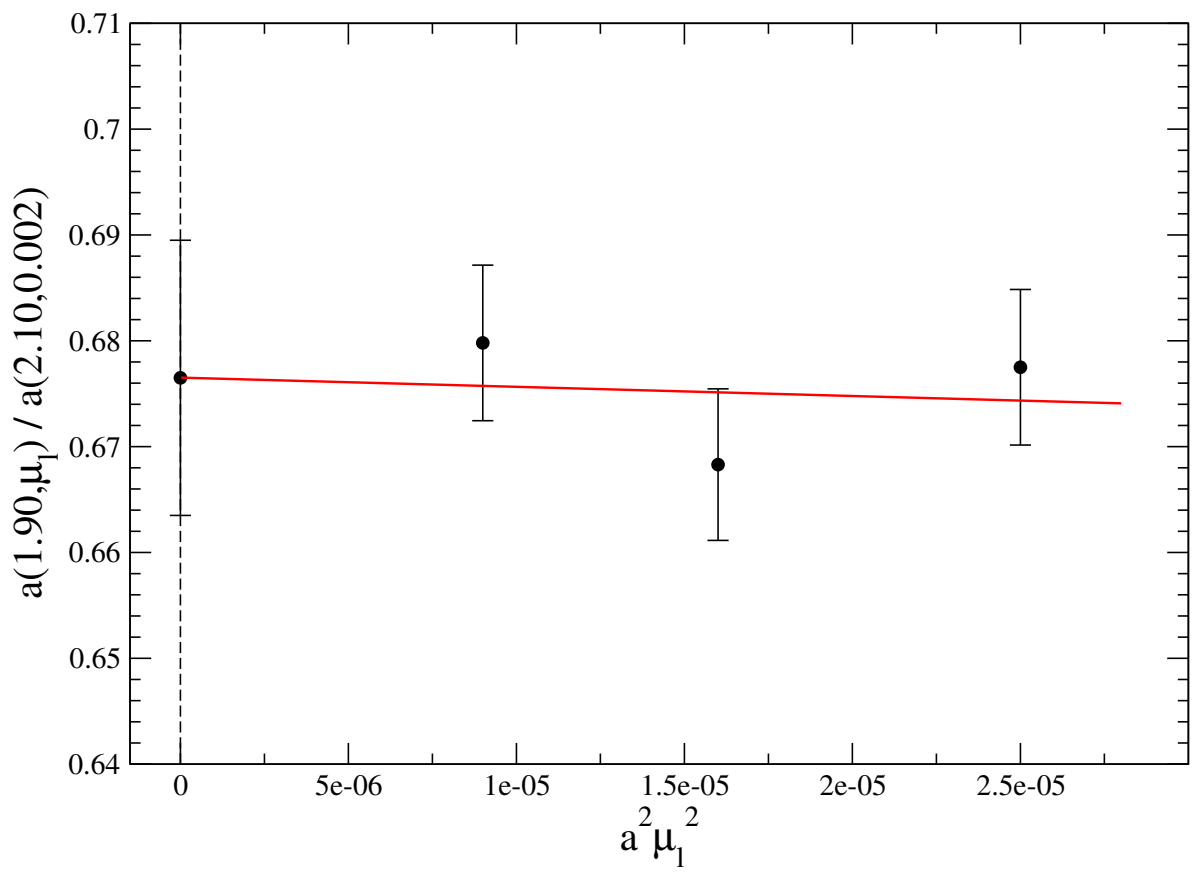

FIG. 6: ratios of lattice spacings from Tab. IV plotted in terms of $a^{2} \mu_{l}^{2}$ which, in the case of fermions simulated with a twisted-mass action at the maximal twist, is proportional to the light quark mass squared. The solid red line corresponds to an extrapolation to the chiral limit according to Eq. (17).
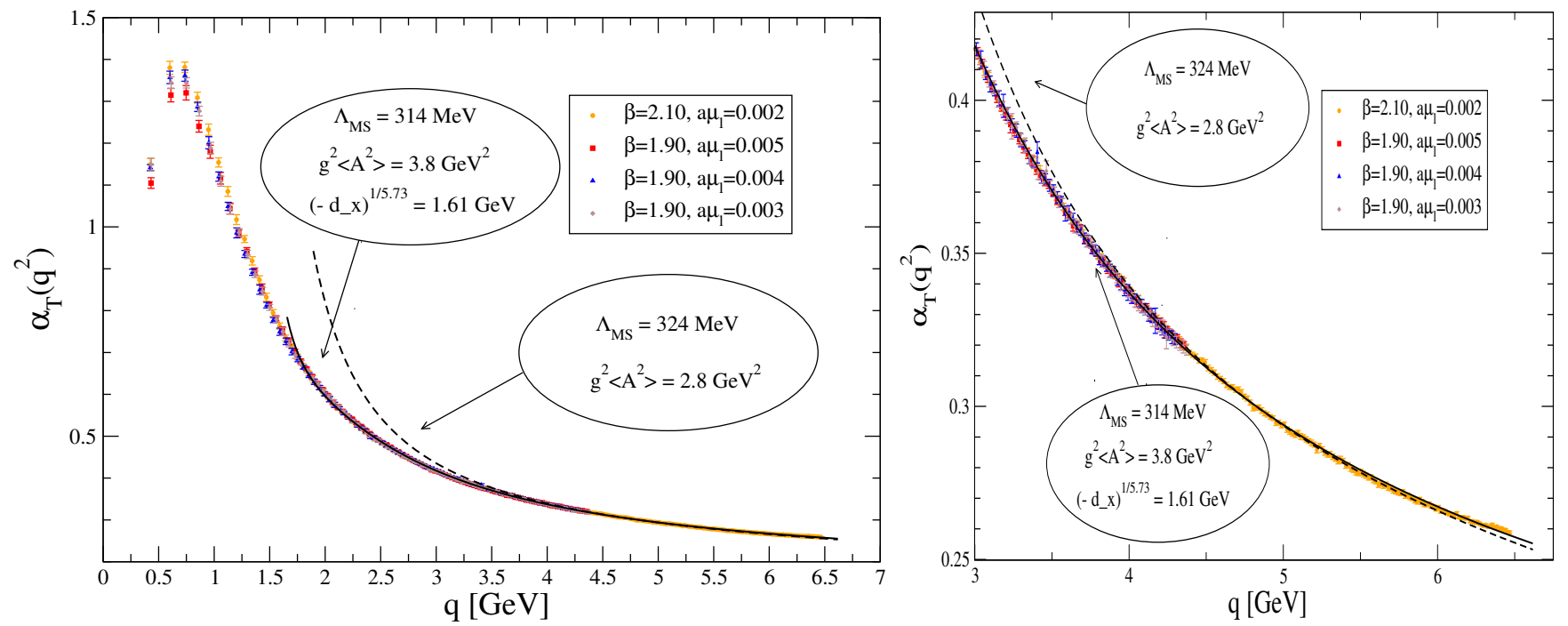

FIG. 7: The Taylor running coupling obtained from the lattice after the appropriate removal of $O(4)$-breaking $(H(4)$ extrapolation) and $O(4)$-invariant artefacts. The curves are fitted to the physical running defined by Eq. 13 with the parameters shown in Tab. V the solid (dashed) black line corresponds to the large (small) fitting window. The right plot is a zoom, focussing on the large momenta region, of the left one.

where we take the central value from the fit including a higher-order power correction and estimate a conservative systematic error (in bold faced characters) with its difference from the value obtained with the fit over the small window, without including the higher-order power. The two other errors quoted in Eq. (20) correspond to the statistical uncertainty from the fit (first) and from the lattice spacing given by Eq. 19 (second).

Then, we can apply the result in Eq. (20) to run the coupling down to the scale of the $\tau$ mass, below the bottom quark mass threshold, and compare the result with the estimate from $\tau$ decays [34, [59, $\alpha_{\overline{\mathrm{MS}}}\left(m_{\tau}^{2}\right)=0.330(14)$. This 
gives, with the $1-\sigma$ propagation given by

$$
\sigma^{2}\left(\alpha_{\overline{\mathrm{MS}}}\left(q^{2}\right)\right)=4 \beta_{\overline{\mathrm{MS}}}^{2}\left(\alpha_{\overline{\mathrm{MS}}}\left(q^{2}\right)\right) \frac{\sigma^{2}\left(\Lambda_{\overline{\mathrm{MS}}}\right)}{\Lambda_{\overline{\mathrm{MS}}}^{2}}
$$

for each error contribution in Eq. (20), the following result at the $\tau$-mass scale:

$$
\alpha_{\overline{\mathrm{MS}}}\left(m_{\tau}^{2}\right)=0.336(4)(8)(6),
$$

in good agreement with the one from $\tau$ decays.

In order to determine $\alpha_{\overline{\mathrm{MS}}}\left(q^{2}\right)$ at the $Z^{0}$ mass scale, we should first run the coupling up to the $\overline{\mathrm{MS}}$ running mass for the bottom quark, $m_{b}$, with $\beta$-coefficients and $\Lambda_{\overline{\mathrm{MS}}}$ estimated for 4 quark flavours, apply next the matching formula [4]:

$$
\alpha_{\overline{\mathrm{MS}}}^{N_{f}=5}\left(m_{b}\right)=\alpha_{\overline{\mathrm{MS}}}^{N_{f}=4}\left(m_{b}\right)\left(1+\sum_{n} c_{n 0}\left(\alpha_{\overline{\mathrm{MS}}}^{N_{f}=4}\left(m_{b}\right)\right)^{n}\right),
$$

where the coefficients $c_{n 0}$ can be found in ref. [56, 57] and finally run from the bottom mass up to the $Z^{0}$ mass scale with $\beta$-coefficients for $N_{f}=5$. We obtain:

$$
\alpha_{\overline{\mathrm{MS}}}\left(m_{Z}^{2}\right)=0.1196(4)(8)(6),
$$

where we have again propagated all the error contributions from Eq. 20p. This result is compatible with the last lattice results averaged by PDG [34, 0.1185(7), and with its world average without including lattice results, 0.1183(12). Eqs. (22 24) update the results of [21] with a much higher statistics for our sample of gauge field configurations which allowed for a more precise result and a more reliable error analysis. The latter is mainly because, in order to be left with stable fits, we did not need to combine data from simulations at different $\beta$ and made no hypothesis on how the lattice artefacts scale for different lattice spacings.

\section{CONCLUSIONS}

The strong coupling renormalized in the MOM Taylor scheme has been obtained from ghost and gluon propagators that have been in their turn computed from several high-statistical samples of gauge field configurations, simulated at two different bare couplings, $\beta$ 's, and four different sets of masses for the degenerate up and down and nondegenerate strange and charm twisted-mass dynamical quark flavors. We thus updated our previous results by performing a significatively improved analysis, which reduced the statistical errors and allowed for a better control of the systematic uncertainties, as those related with higher-order OPE corrections and lattice artefacts. Furthermore, we took full account within the estimate of statistical errors of the matching procedure to determine the ratio of different lattice spacings and of the chiral extrapolation to the physical point for their absolute "calibration". Last but not least, we avoided to assume the constancy of the coefficient $c_{a 2 p 2}$ for the $O(4)$-invariant lattice artefacts and indeed found it to happen consistently with the approximation of neglecting higher-order artefacts.

As a result of our improved analysis, we confirmed the need to include nonperturbative corrections in order to describe the lattice data for the MOM Taylor strong coupling for momenta below $7 \mathrm{GeV}$. After dealing properly with lattice artefacts, the Wilson coefficient for the leading OPE power correction, known at the $\mathcal{O}\left(\alpha^{4}\right)$-order, is found to account for data with momenta roughly above $4 \mathrm{GeV}$ and, along with an effective higher-order power correction, above $\simeq 2 \mathrm{GeV}$. The value of $\Lambda_{\overline{\mathrm{MS}}}$, for $N_{f}=4$, has been obtained from the running of the Taylor strong coupling and used then to estimate the $\overline{\mathrm{MS}}$ coupling at the $\tau$-mass scale and, properly handling the transition from $N_{f}=4$ to $N_{f}=5$, at the $Z^{0}$-mass scale. In both cases, our estimates agree with the "world average" results the Particle Data Group provides. Therefore, with the lattice-regularized QCD action we used, the pion mass and decay constant have been taken as the physical scales to size the strong coupling in the appropriate momentum window, roughly from 2 to $7 \mathrm{GeV}$. As far as perturbative QCD is, in its turn, applied to run the coupling from those momenta up to $Z^{0}$-mass scale, a main conclusion of this work is that $Q C D$ is successfully bridging from the low-momentum pion sector up to the very $U V$ domain for the strong interactions.

\section{Acknowledgements}

We are particularly indebted to A. Le Yaouanc, J. P. Leroy and J. Micheli for participating in many fruitful discussions at the preliminar stages of this work. We thank the support of Spanish MICINN FPA2011-23781 research project 
and the IN2P3 (CNRS-Lyon), IDRIS (CNRS-Orsay), TGCC (Bruyes-Le-Chatel), CINES (Montpellier). K.Petrov is part of P2IO Laboratory of Excellence.

\section{Appendix A: Hypercubic $H(4)$-extrapolation}

The first kind of artefacts that can be systematically cured [46, 48] are those due to the breaking of the rotational symmetry of the Euclidean space-time when using an hypercubic lattice, where this symmetry is restricted to the discrete $H(4)$ isometry group. Let us consider a general dimensionless lattice quantity $Q\left(a q_{\mu}\right)$ that will be conveniently averaged over every orbit of the group $H(4)$. In general several orbits of $H(4)$ correspond to one value of $q^{2}$. Defining the $H(4)$ invariants

$$
q^{[4]}=\sum_{\mu=1}^{4} q_{\mu}^{4} \quad q^{[6]}=\sum_{\mu=1}^{6} q_{\mu}^{6},
$$

it happens that the orbits of $H(4)$ are labelled ${ }^{6}$ by the set $q^{2}, a^{2} q^{[4]}, a^{4} q^{[6]}$. We can thus define the quantity $Q\left(a q_{\mu}\right)$ averaged over $H(4)$ as

$$
Q\left(a^{2} q^{2}, a^{4} q^{[4]}, a^{6} q^{[6]}\right) .
$$

In the continuum limit the effect of $a^{2} q^{[4]}, a^{4} q^{[6]}$ vanishes, as indeed happens for a free lattice propagator:

$$
\frac{1}{\frac{1}{a^{2}} \sum_{\mu}\left(1-\cos \left(a q_{\mu}\right)\right)^{2}}=\frac{1}{q^{2}}\left(1+\frac{1}{12} \frac{a^{2} q^{[4]}}{q^{2}}+\cdots\right) \text {. }
$$

If the lattice spacing is small enough such that $\epsilon=a^{2} q^{[4]} / q^{2} \ll 1$, the dimensionless lattice correlation function defined in Eq. A2 can be expanded in powers of $\epsilon$ :

$$
Q\left(a^{2} q^{2}, a^{4} q^{[4]}, a^{6} q^{[6]}\right)=\widehat{Q}\left(a^{2} q^{2}\right)+\left.\frac{d Q}{d \epsilon}\right|_{\epsilon=0} a^{2} \frac{q^{[4]}}{q^{2}}+\cdots
$$

Then, one can fit the coefficient $d Q / d \epsilon$ from the whole set of orbits sharing the same $q^{2}$ and get $\widehat{Q}$, the extrapolated value of $Q$ free from $H(4)$ artefacts. If we further assume that the coefficient

$$
R\left(a^{2} q^{2}\right)=\left.\frac{d Q}{d \epsilon}\right|_{\epsilon=0}
$$

has a smooth dependence on $a^{2} q^{2}$ over a given momentum window, we can expand it as $R=R_{0}+R_{1} a^{2} q^{2}$ and make a global fit in the whole momentum window to extract the extrapolated value of $Q$ for any momenta $q$. The contribution from the term $a^{4} q^{[6]} / q^{2}$ has been also neglected. The very good matching for the estimates of $\alpha_{T}$ from simulations with different lattice spacings we have obtained (and that can be seen in Fig. 7) proves the latter to be a good approximation.

On the other hand, the continuum limit (and the full $O(4)$ rotational invariance) has to be reached when $\epsilon=$ $a^{2} q^{[4]} / q^{2} \rightarrow a^{2} q^{2} / 2 \rightarrow 0$. Then, we could have also expanded the attice correlation function defined in Eq. A2 as

$$
Q\left(a^{2} q^{2}, a^{4} q^{[4]}, a^{6} q^{[6]}\right)=\widehat{Q^{\prime}}\left(a^{2} q^{2}\right)+\left.\frac{d Q}{d \epsilon}\right|_{\epsilon=0}\left(a^{2} \frac{q^{[4]}}{q^{2}}-\frac{a^{2} q^{2}}{2}\right)+\cdots
$$

Anyhow, $\widehat{Q}$ in Eq. A4 only differs from $\widehat{Q^{\prime}}$ in Eq. A5 by a term proportional to $a^{2} q^{2}$, not breaking $O(4)$ symmetry, that will be furtherly dropped in the next step (see Eq. (8)), when curing the remaining $O(4)$-invariant artefacts. Then, we have chosen to apply Eq. (A4) that, when $\alpha_{T}$ replaces $Q$, reads as Eq. (7).

\footnotetext{
${ }^{6}$ On totally general grounds, any $H(4)$-invariant polynome can be written only in terms of the four invariants $q^{[2 i]}$ with $i=1,2,3,4$ [46, 48 . As a consequence of the upper cut for momenta, the first three of these invariants suffice to label all the orbits we deal with and hence any presumed dependence on $q^{[8]}$ is neglected.
} 
It is finally worthwile to mention that we considered in this work anisotropic lattice of the type $L^{3} \mathrm{x} T$, with $T=2 L$. This finite volume effect reduces the $H(4)$ lattice symmetry to $H(3)$. Deviations from $H(4)$ are to be expected in the long-distance physics. But ultraviolet physics should not be affected. As far as we are interested in the high-momentum regime, we will assume the previous treatement of the lattice artefacts to be valid.

[1] M. Luscher, R. Sommer, P. Weisz, and U. Wolff, Nucl.Phys. B413, 481 (1994), hep-lat/9309005.

[2] G. M. de Divitiis, R. Frezzotti, M. Guagnelli, and R. Petronzio, Nucl. Phys. B433, 390 (1995), hep-lat/9407028.

[3] M. Della Morte et al. (ALPHA), Nucl. Phys. B713, 378 (2005), hep-lat/0411025.

[4] S. Aoki et al. (PACS-CS), JHEP 10, 053 (2009), 0906.3906.

[5] S. Booth et al. (UKQCD Collaboration), Phys.Lett. B294, 385 (1992), hep-lat/9209008.

[6] N. Brambilla, X. Garcia i Tormo, J. Soto, and A. Vairo, Phys.Rev.Lett. 105, 212001 (2010), 1006.2066.

[7] M. Gockeler et al., Phys. Rev. D73, 014513 (2006), hep-ph/0502212.

[8] Q. Mason et al. (HPQCD), Phys. Rev. Lett. 95, 052002 (2005), hep-lat/0503005.

[9] K. Maltman, D. Leinweber, P. Moran, and A. Sternbeck, Phys. Rev. D78, 114504 (2008), 0807.2020.

[10] C. Davies et al. (HPQCD Collaboration), Phys.Rev. D78, 114507 (2008), 0807.1687.

[11] I. Allison et al. (HPQCD Collaboration), Phys.Rev. D78, 054513 (2008), 0805.2999.

[12] C. McNeile, C. Davies, E. Follana, K. Hornbostel, and G. Lepage, Phys.Rev. D82, 034512 (2010), 1004.4285.

[13] K. Jansen, M. Petschlies, and C. Urbach, PoS LATTICE2011, 234 (2011), 1111.5252.

[14] B. Alles, D. Henty, H. Panagopoulos, C. Parrinello, C. Pittori, et al., Nucl.Phys. B502, 325 (1997), hep-lat/9605033.

[15] P. Boucaud, J. Leroy, J. Micheli, O. Pène, and C. Roiesnel, JHEP 9810, 017 (1998), hep-ph/9810322.

[16] P. Boucaud et al., JHEP 04, 006 (2000), hep-ph/0003020.

[17] P. Boucaud, A. Le Yaouanc, J. Leroy, J. Micheli, O. Pène, and J. Rodríguez-Quintero, Phys.Lett. B493, 315 (2000), hep-ph/0008043.

[18] P. Boucaud, A. Le Yaouanc, J. Leroy, J. Micheli, O. Pène, and J. Rodríguez-Quintero, Phys.Rev. D63, 114003 (2001), hep-ph/0101302.

[19] P. Boucaud et al., JHEP 01, 046 (2002), hep-ph/0107278.

[20] A. Sternbeck et al., PoS LAT2007, 256 (2007), 0710.2965.

[21] B. Blossier, P. Boucaud, M. Brinet, F. De Soto, X. Du, et al., Phys.Rev.Lett. 108, 262002 (2012), 1201.5770.

[22] B. Blossier, P. Boucaud, M. Brinet, F. De Soto, X. Du, et al., Phys.Rev. D85, 034503 (2012), 1110.5829.

[23] P. Boucaud, F. De Soto, J. Leroy, A. Le Yaouanc, J. Micheli, O. Pène, and J. Rodríguez-Quintero, Phys.Rev. D79, 014508 (2009), 0811.2059.

[24] J. Rodriguez-Quintero et al., PoS QCD-TNT09, 039 (2009), 0911.0568.

[25] B. Blossier et al. (ETM), Phys. Rev. D82, 034510 (2010), 1005.5290.

[26] B. Blossier et al. (ETM), PoS LATTICE2010, 227 (2010), 1010.5724.

[27] A. Sternbeck et al., PoS LAT2009, 210 (2009), 1003.1585.

[28] A. Sternbeck, K. Maltman, M. Muller-Preussker, and L. von Smekal, PoS LATTICE2012, 243 (2012), 1212.2039.

[29] F. V. Gubarev and V. I. Zakharov, Phys. Lett. B501, 28 (2001), hep-ph/0010096.

[30] D. Dudal, R. Sobreiro, S. Sorella, and H. Verschelde, Phys.Rev. D72, 014016 (2005), hep-th/0502183.

[31] P. Boucaud et al., Phys. Rev. D66, 034504 (2002), hep-ph/0203119.

[32] M. A. Shifman, A. Vainshtein, and V. I. Zakharov, Nucl.Phys. B147, 385 (1979).

[33] M. A. Shifman, A. Vainshtein, and V. I. Zakharov, Nucl.Phys. B147, 448 (1979).

[34] J. Beringer et al. (Particle Data Group), Phys.Rev. D86, 010001 (2012).

[35] B. Blossier, P. Boucaud, M. Brinet, F. De Soto, V. Morenas, et al., Phys. Rev. D 87, 074033 (2013), 1301.7593.

[36] J. Taylor, Nucl.Phys. B33, 436 (1971).

[37] L. von Smekal, A. Hauck, and R. Alkofer, Annals Phys. 267, 1 (1998), hep-ph/9707327.

[38] A. Aguilar, D. Binosi, and J. Papavassiliou, PoS LC2008, 050 (2008), 0810.2333.

[39] D. Binosi and J. Papavassiliou, Phys.Rept. 479, 1 (2009), 0909.2536.

[40] A. C. Aguilar, D. Binosi, J. Papavassiliou, and J. Rodriguez-Quintero, Phys. Rev. D80, 085018 (2009), 0906.2633.

[41] A. Aguilar, D. Binosi, and J. Papavassiliou, JHEP 1007, 002 (2010), 1004.1105.

[42] J. A. Gracey, Phys. Lett. B552, 101 (2003), hep-th/0211144.

[43] K. Chetyrkin and A. Maier, JHEP 1001, 092 (2010), 0911.0594.

[44] K. Nakamura et al. (Particle Data Group), J. Phys. G37, 075021 (2010).

[45] K. Chetyrkin and A. Retey (2000), hep-ph/0007088.

[46] D. Becirevic, P. Boucaud, J. Leroy, J. Micheli, O. Pene, J. Rodriguez-Quintero, and C. Roiesnel, Phys. Rev. D60, 094509 (1999), hep-ph/9903364.

[47] D. Becirevic, P. Boucaud, J. Leroy, J. Micheli, O. Pene, J. Rodriguez-Quintero, and C. Roiesnel, Phys. Rev. D61, 114508 (2000), hep-ph/9910204.

[48] F. de Soto and C. Roiesnel, JHEP 0709, 007 (2007), 0705.3523.

[49] P. Boucaud, F. de Soto, J. Leroy, A. Le Yaouanc, J. Micheli, et al., Phys.Lett. B575, 256 (2003), hep-lat/0307026.

[50] P. Boucaud, F. de Soto, J. Leroy, A. Le Yaouanc, J. Micheli, et al., Phys.Rev. D74, 034505 (2006), hep-lat/0504017. 
[51] B. Blossier et al., Phys. Rev. D83, 074506 (2011), 1011.2414.

[52] R. Frezzotti, P. A. Grassi, S. Sint, and P. Weisz (Alpha), JHEP 08, 058 (2001), hep-lat/0101001.

[53] R. Baron, P. Boucaud, J. Carbonell, A. Deuzeman, V. Drach, et al. (ETM Collaboration), JHEP 1006, 111 (2010), 1004.5284

[54] R. Baron, B. Blossier, P. Boucaud, J. Carbonell, A. Deuzeman, et al. (ETM Collaboration), PoS LATTICE2010, 123 (2010), 1101.0518.

[55] R. Frezzotti and G. C. Rossi, Nucl. Phys. Proc. Suppl. 128, 193 (2004), hep-lat/0311008.

[56] K. Chetyrkin, J. H. Kuhn, and C. Sturm, Nucl.Phys. B744, 121 (2006), hep-ph/0512060.

[57] Y. Schroder and M. Steinhauser, JHEP 0601, 051 (2006), hep-ph/0512058.

[58] N. Carrasco, P. Dimopoulos, R. Frezzotti, P. Lami, V. Lubicz, et al. (2013), 1311.2793.

[59] S. Bethke, A. H. Hoang, S. Kluth, J. Schieck, I. W. Stewart, et al. (2011), 1110.0016 [hep-ph]. 